\title{
Elastoplastic buckling and collapse of spherical shells under combined loadings
}

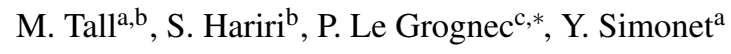 \\ ${ }^{a}$ CETIM - Centre Technique des Industries Mécaniques, 5 avenue Félix Louat - BP 80067, F-60304 Senlis Cedex, France \\ ${ }^{b}$ Mines Douai, Polymers and Composites Technology \& Mechanical Engineering Department, 941 rue Charles Bourseul - CS 10838, F-59508 \\ Douai Cedex, France \\ ${ }^{c}$ ENSTA Bretagne, FRE CNRS 3744, IRDL, F-29200 Brest, France
}

\begin{abstract}
In this paper, the buckling behavior of a complete spherical shell under external pressure and of a half-sphere under torsion are first investigated. Several parametric studies are performed, which rely on the European Recommendations framework (ECCS text), so as to identify the different key parameters of the corresponding buckling capacity curves and lead up to both a qualitative and quantitative discussion about the imperfection sensitivity of such structures under each fundamental load. The related results allow us to completely describe the buckling behavior of a spherical shell under combined external pressure and circumferential shear by means of interactive buckling curves, without the need of many supplementary computations for any arbitrary combined loading case.
\end{abstract}

Keywords: Spherical shells, Buckling/Post-buckling, Elastoplasticity, External pressure/Torsion, Interaction curves, Geometric imperfections.

\section{Introduction}

Shell structures are widely used in industrial applications, such as pressure vessels, transportation facilities and buildings. However, the economic interest of such thin structures is counteracted by their weaknesses in terms of mechanical strength, when compared to solid structures. In the particular case of pressure vessels submitted to compressive loadings, buckling is one of the main failure modes and often leads to significant material and human risks. It has been therefore the subject of many studies for more than a century, all the more so as large discrepancies between theoretical predictions and experiments are commonly observed (since the pioneering works of Lorenz, Timoshenko and Southwell in the case of a cylindrical shell under axial compression). It is not so long ago that this phenomenon has been explained, thanks to the contributions of von Karman and Tsien, and then to the decisive one of Koiter [1]. The latter built the foundations of the post-buckling analysis and proved that, contrary to beams and plates, shells are generally shown to be very imperfection-sensitive. Unsure of the precise nature and amplitude of the initial defects, and for efficiency purposes, the most current design method used in shell buckling relies typically on a combination of

\footnotetext{
* Corresponding author

Email addresses: mamadou.tall@cetim.fr (M. Tall), said.hariri@imt-lille-douai.fr (S. Hariri), philippe. le_grognec@ensta-bretagne.fr (P. Le Grognec), yves . simonet@cetim.fr (Y. Simonet) 
theoretical results on perfect structures and the use of knock-down factors, which are supposed to account for possible geometric or material imperfections, or loading/boundary condition effects.

In the case of a complete spherical shell under external pressure, Zoelly [2] was the first to derive the elastic critical buckling pressure under the assumptions of small pre-critical displacements and axisymmetric modal deformations:

$$
P_{c r_{(\text {Zoelly })}}=\frac{2 E}{\sqrt{3\left(1-v^{2}\right)}}\left(\frac{t}{R}\right)^{2}
$$

where $E$ and $v$ stand for the Young's modulus and the Poisson's ratio, respectively, $t$ is the thickness and $R$ the radius of the spherical shell. Later, Van der Neut [3] demonstrated that the aforementioned critical pressure was still relevant in the general case of a non-axisymmetric mode and that there were several modes (of both types) associated with the same critical buckling load. In the 1960's, Hutchinson [4] applied Koiter's general theory to the spherical shell problem, using the shallow shell kinematics hypotheses, so as to study the effects induced by the interaction between two or three multiple modes. He showed that the presence of several modes at the same critical load had a deleterious effect on the buckling response of the spherical shell. Finally, by using an axisymmetric imperfection pattern, he investigated the sensitivity of such a structure to initial geometric imperfections, and determined a lower bound for the corresponding critical pressure. Subsequently, numerous theoretical [4-7], numerical [8-10] and experimental [11-14] studies have shown that spherical shells under external pressure, in a similar manner to cylindrical shells under axial compression, are among the most imperfection-sensitive structures. Therefore, the challenge was to find the "worst" or at least the most "stimulating" imperfection pattern which would lead to a safe design. Deml and Wunderlich [15] developed a fully non-linear finite element model, without any approximation, in order to better estimate the minimum buckling load. The key point of the method was to consider the imperfection as a supplementary entry into the governing equations. Then considering the resulting extended system, the authors obtained directly the "worst" imperfection pattern together with the corresponding critical load. In practice, they found that, for a spherical shell under external pressure, the "worst" imperfection pattern was a localized dimple. Nevertheless, Wunderlich and Albertin [10] showed that the load carrying capacity of a spherical cap was similarly affected by an imperfection in the form of a single dimple and an imperfection defined in terms of the first linear eigenmode. Lately, Błażejewski and Marcinowski [16] retrieved the full capacity curves of a clamped spherical cap under external pressure, in the sense of the European Recommendations framework, by exploring different imperfection patterns. More recently, Jiménez et al. [17] and Lee et al. [18] conducted experimental tests and numerical computations on spherical shells in the presence of relatively precise engineered geometric imperfection patterns. Their outcomes led to the definition of a knock-down factor.

Dealing now with the buckling of spherical shells under shear loads, to the best knowledge of the authors, very few publications can be found in the literature. Yinyi [19] used differential geometry analysis and a Galerkin type procedure to retrieve and solve the exact equations for the buckling of a spherical shell under circumferential shear. The author pointed out that, in terms of stability, deep spherical shells $\left(30^{\circ}<\varphi<90^{\circ}\right.$, where $\varphi$ is the semi-angle of the spherical cap) are stronger than shallow spherical shells. Mow and Sadowski [20] studied the buckling behavior of 
a spherical shell subjected to a moment torque at the poles. The results showed that "oblong-elliptic" dimples may appear as buckling initiates. The authors gave an heuristic solution for the critical moment torque $T_{c r}$ and the size of the minor semi-axis $b$ of the elliptic dimples. Also, the buckling response under combined loading has been outlined by the same authors. Among their noticeable results, the authors determined the so-called interactive buckling curve for a spherical shell subjected to a moment torque and external (or internal) pressure in the linear elastic range.

In practice, as emphasized by Schmidt and Winterstetter [21], a combination of such fundamental loads (external pressure, torsion, ...) does not necessary induce a simple fundamental membrane stress state in the pre-critical range, but a combination of them. As this combination may be extremely complex, one has to perform a design interaction check for an efficient and reliable buckling analysis. In the literature, the buckling phenomenon under combined loading has been extensively addressed for cylindrical shells [22--24], conical shells [25-27] and also for spherical shells under external pressure and point loads [28, 29]. Conversely, except the one from Mow and Sadowski [20] previously mentioned, such an interaction analysis has not been achieved so far in the case of a spherical shell under both external pressure and circumferential shear.

\section{EN 1993-1-6 and ECCS text framework}

The ECCS text provides an extensive commentary of the shell Eurocode EN 1993-1-6 [30] and extends the coverage of the latter standard to many additional specific problems. In the sequel, unless otherwise stated, the notations of the two formalisms are deemed to be equivalent. The ECCS text defines different "computation levels" in order to facilitate communication between engineers and researchers (see Table 1 ).

\begin{tabular}{ll}
\hline LBA & $\begin{array}{l}\text { Linear Bifurcation Analysis (linear elastic eigenvalue computation performed on the } \\
\text { perfect structure), } R_{L B A}\end{array}$ \\
MNA & $\begin{array}{l}\text { Materially Non-linear Analysis (small displacement theory, non-elastic material behav- } \\
\text { ior), } R_{M N A} \\
\text { Geometrically Non-linear Analysis of the perfect structure (elastic material behavior, } \\
\text { search for bifurcation and snap-through points), } R_{G N A}\end{array}$ \\
GNA & $\begin{array}{l}\text { Geometrically and Materially Non-linear Analysis of the perfect structure (non-elastic } \\
\text { material behavior, search for bifurcation and snap-through points), } R_{G M N A}\end{array}$ \\
GNIA & $\begin{array}{l}\text { Geometrically Non-linear Analysis of the Imperfect structure (elastic material behavior, } \\
\text { introducing geometric substitute imperfections), } R_{G N I A} \\
\text { Geometrically and Materially Non-linear Analysis of the Imperfect structure (non- } \\
\text { elastic material behavior, introducing geometric substitute imperfections), } R_{G M N I A}\end{array}$ \\
\hline
\end{tabular}

Table 1: "Computation levels" according to EN 1993-1-6 [31] and ECCS text [30] and the corresponding resistance $R_{i}$ for each analysis (adapted from [21])

Each of the analyses presented in Table 1 gives useful information about the global behavior of a shell structure. In shell structures, buckling and plasticity can be wisely considered as the main failure phenomena. The EN 1993-1-6 
strength:

$$
\chi=\frac{R_{G M N I A}}{R_{M N A}}
$$

is plotted against the relative slenderness of the structure:

$$
\lambda=\sqrt{\frac{R_{M N A}}{R_{L B A}}}
$$

which governs the assessment of the relative importance of buckling and plasticity in the final collapse of the shell.

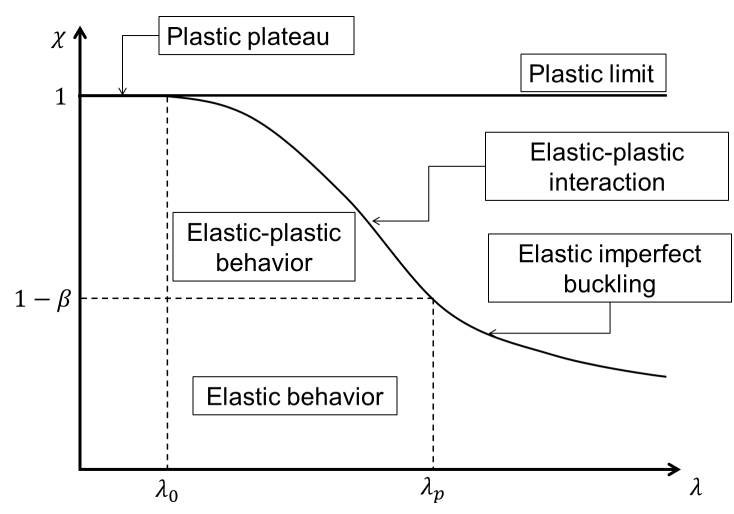

(a) Traditional capacity curve

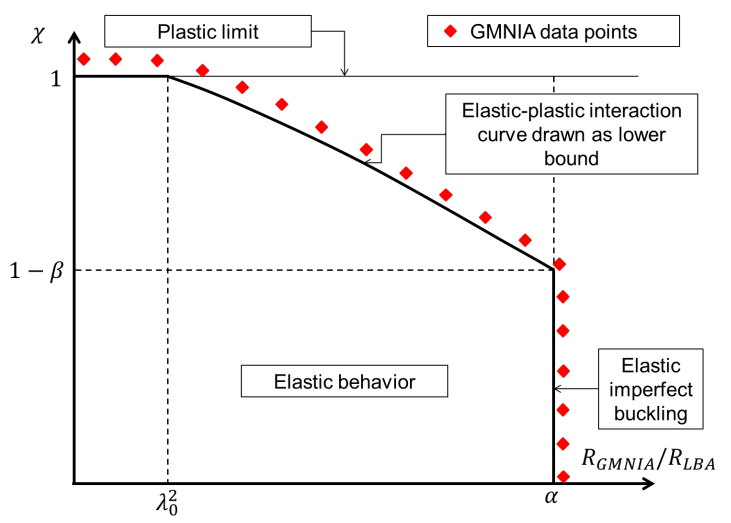

(b) Modified capacity curve [32-34]

Figure 1: Capacity curves of EN 1993-1-6 [31] and ECCS [30]

From many numerical computations in different configurations, one obtains curves like the one in Figure 1(a) (one for each imperfection level), which may be approximated by the following expressions:

$$
\begin{cases}\chi=1 & \text { when } \lambda \leq \lambda_{0} \\ \chi=1-\beta\left(\frac{\lambda-\lambda_{0}}{\lambda_{p}-\lambda_{0}}\right)^{\eta} & \text { when } \lambda_{0} \leq \lambda \leq \lambda_{p} \\ \chi=\frac{\alpha}{\lambda^{2}} & \text { when } \lambda_{p} \leq \lambda\end{cases}
$$

where:

$$
\lambda_{p}=\sqrt{\frac{\alpha}{1-\beta}}
$$

In (4), one can observe that the so-called buckling capacity curves are totally defined once the four key parameters, namely $\alpha, \beta, \eta$ and $\lambda_{0}$, are known. These parameters are not arbitrary and each of them is related to a specific aspect concerning the buckling phenomenon: under elastic conditions, the elastic imperfection factor $\alpha$ describes the loss of strength due to geometric non-linearities and geometric imperfections; the load level at which yielding first affects the buckling strength is captured by the plastic range factor $\beta$; the slenderness at which the plastic reference resistance is exceeded as a result of strain hardening is given by the squash limit relative slenderness $\lambda_{0}$; and finally, the shape of the 
elastic-plastic part of the curve is given by the interaction exponent $\eta$.

In practice, experience shows that the use of the traditional capacity curves [Figure 1(a) for the determination of the key parameters defined above can be very challenging. Thus, in the sequel, use will be made of Rotter's modified capacity curves [32-34] which rely on an alternative representation (Figure 1(b)] and lead up more easily to the different key parameters. These curves are obtained by varying the yield stress of the material, for a given geometric ratio and imperfection level.

\section{Scope and outline of the paper}

The EN 1993-1-6 [31] and the ECCS text [30] give three provisions for the design of shell structures: hand calculations, MNA/LBA procedure and fully numerical GMNIA analyses. However, hand calculations are generally too simplified to achieve reliable results in the case of complicated shell structures and the GMNIA analyses are far too onerous to be economically viable. The half-way house MNA/LBA procedure overcomes those reserves. The method is based on the idea that a numerical software (or analytical expressions, when available) can be used to estimate the elastic bifurcation reference load $R_{L B A}$ and the plastic reference limit load $R_{M N A}$ in order to determine the relative slenderness of the structure $\lambda$. Then, once the buckling capacity curves are identified for the shell buckling problem in hand with similar characteristics (in terms of imperfection level), the actual resistance can be assessed easily and thus the buckling design is straightforward. The main disadvantage of this method stems from the fact that the capacity curves are related to a specific shell configuration which is supposed, a priori, to be unique. Therefore, a question naturally arises: how to ensure that the parameters used in the MNA/LBA procedure are approximately correct?

The objective of this paper is first to extract the capacity curves of spherical shells under two fundamental loading cases (external pressure and torsion) by using the various "computation levels" (LBA, MNA, GNA, GNIA and GMNIA) of the ECCS Recommendations. On one hand, it is noteworthy that, for the spherical shell under external pressure, those capacity curves are already implemented in the ECCS text thanks to the extensive parametric studies of Wunderlich and Albertin [10, 35]. However, parameters $\beta$ and $\eta$ were chosen conservatively as constants, namely $\beta=0.70$ and $\eta=1.0$. The results of the large parametric studies performed in the present study and the adoption of the assumptions of Doerich and Rotter [36] concerning $\eta$ (which is supposed to vary linearly with the slenderness) allow us to define more accurate values for parameters $\beta$ and $\eta$ (see Section 5.2). On the other hand, the definition of the full capacity curves for the spherical shell under circumferential shear is, to the best knowledge of the authors, entirely new (see Section 6.2). Finally, the buckling behavior of a spherical shell under combined loadings (external pressure and circumferential shear) will be explored using the so-called interactive buckling curves [10, 21, 32] (see Section77).

\section{Finite Element Model}

In the first loading case of external pressure, the numerical computations are performed on a complete spherical shell so as to capture all the possible axisymmetric and non-axisymmetric eigenmodes. On the contrary, because of 


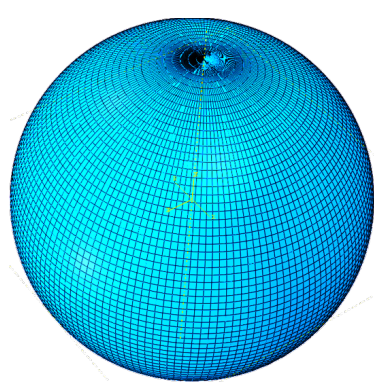

(a) Axisymmetric mesh with singular points

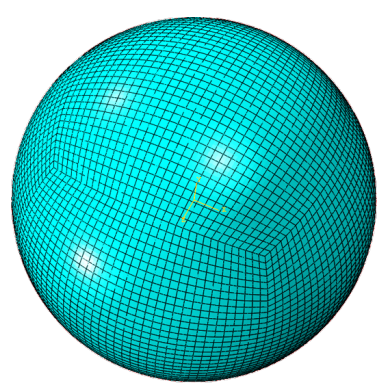

(b) Regular quadrangular mesh

Figure 2: Different meshes for the complete spherical shell under external pressure

the numerous parametric computations required, only one half of the spherical shell is represented in both cases of torsion and combined loading (external pressure and circumferential shear), the remaining half being taken into account by the relevant symmetry conditions. Initially, two types of mesh were used for the finite element calculations: an axisymmetric mesh (Figure 2(a) with respect to an arbitrary diameter of the spherical shell giving rise to two singular points localized at the poles, and a more regular quadrangular mesh Figure 2(b) $^{1}$ created using a special FORTRAN ${ }^{(C)}$ routine. In most cases, use is made of the commercial Finite Element (FE) software package ABAQUS [37]. The non-linear analyses are carried out using the modified Riks method [38] implemented in ABAQUS. The case of a perfect shell (without geometric imperfections) is handled by a specific bespoke FE program [39]. It is developed in a total Lagrangian formulation framework, and allows one to detect true elastic/plastic bifurcation points and proceed automatically onto the post-critical branches, by means of appropriate branch-switching techniques (without the need for initial imperfections). It has already been successfully applied in the context of cylindrical shells under axial compression (see [39] for more details).

All the results obtained in this paper, unless otherwise specified, concern a single geometry (termed as "reference" configuration) whose material and geometric properties are given in Table 2. Eight-node (quadratic) thin shell elements, with five degrees of freedom per node and reduced integration (S8R5 elements in ABAQUS), are predominantly used.

The circumferential shear load is defined by the following surface densities [19] as projected in the spherical coordinate system $\left(\overrightarrow{e_{r}}, \overrightarrow{e_{\theta}}, \overrightarrow{e_{\varphi}}\right)$ (see Figure 3 ):

$$
\left\{\begin{array}{l}
Q_{1}=0 \\
Q_{2}=\tau_{0} \cdot \sin \left(\frac{\pi \varphi}{\alpha}\right) \\
Q_{3}=0
\end{array}\right.
$$

where $\tau_{0}$ is the shear load amplitude, $\alpha$ is the semi-angle of the spherical cap (for the complete spherical shell, $\alpha=90^{\circ}$ ) and $\varphi$ is the azimuthal angle according to the spherical coordinate system (see Figure 3 . The user subroutine capabilities of ABAQUS make it possible to implement easily such a distributed loading.

As stated in Section 1, the problem of a spherical shell under external pressure, similarly to the one of a cylindrical shell under axial compression, reveals multiple modes for each critical value, especially at the minimum one [1]. All 


\begin{tabular}{c|c|c}
\hline Young's modulus & $E$ & $210 \mathrm{GPa}$ \\
Poisson's ratio & $v$ & 0.3 \\
Radius & $R$ & $500 \mathrm{~mm}$ \\
Thickness & $t$ & $4 \mathrm{~mm}$ \\
Yield stress & $\sigma_{y}$ & $235 \mathrm{MPa}$ \\
\hline
\end{tabular}

Table 2: Material and geometric parameters for the "reference" model
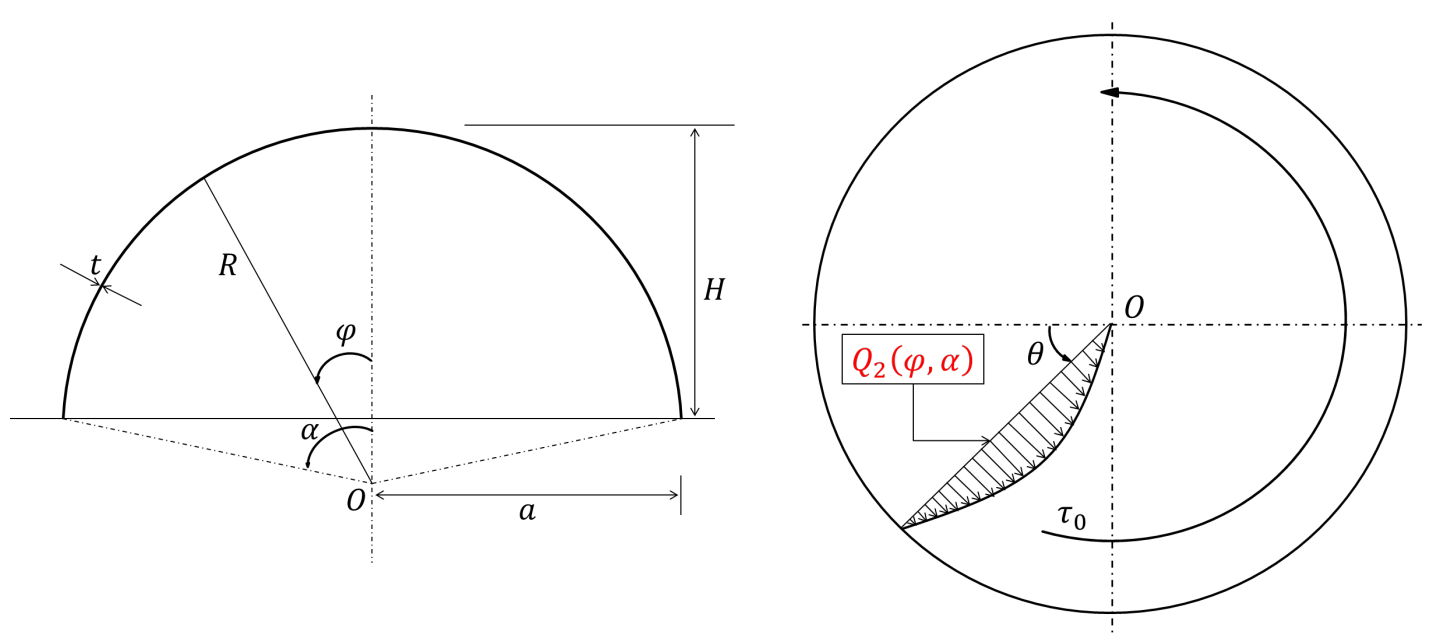

Figure 3: Geometry and loading for the spherical shell under circumferential shear (adapted from [19])

these buckling modes may therefore interact in the vicinity of the bifurcation point (due to non-linearities), leading thus to a high imperfection sensitivity and a significant drop of the carrying capacity of the shell. Henceforth, it is well established that even a small imperfection can be deleterious for an imperfection-sensitive structure [5, 34, 40]. In the following analyses, the imperfection sensitivity of the spherical shell will be thus investigated by using the eigenmode associated with the lowest critical buckling load, obtained by a Linear Bifurcation Analysis (LBA), as the initial imperfection pattern in all the computations related to the imperfect structure.

\section{Spherical shell under external pressure}

\subsection{Numerical results and discussions}

\subsubsection{Linear Bifurcation Analysis (LBA)}

First, an elastic linearized buckling analysis is performed using ABAQUS, so as to identify the first critical pressure and the corresponding eigenmodes. The numerical results show that the critical modes are highly mesh-dependent. Indeed, with the axisymmetric mesh, most of the eigenmodes are axisymmetric (see Figures 4(a) and 4(b)), while for the regular mesh, the first eigenmode displays a reticulated pattern with regular waves in both directions (see Figure 4(c) . Notwithstanding the difference observed in the buckling mode patterns, the critical buckling pressures appear to be similar for the two mesh types and clustered in each case (more than the first fifty modes correspond to a one and only critical value). This particular feature is a primal characteristic of imperfection-sensitive structures, as 
shown in Koiter's initial post-buckling theory. Thus, a rapid decrease of the bearing capacity of the shell has to be expected after the buckling load.

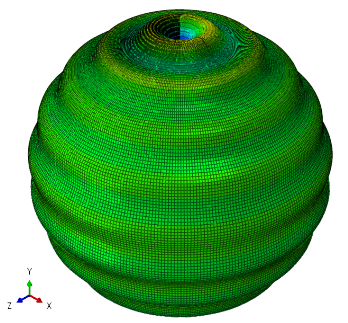

(a) Mode 1 with axisymmetric mesh

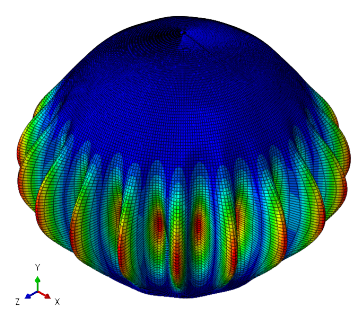

(b) Mode 5 with axisymmetric mesh

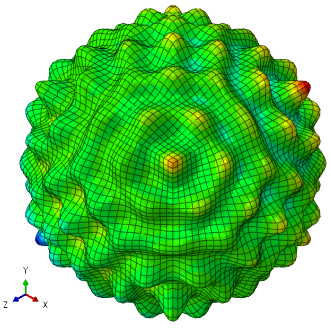

(c) Mode 1 with regular mesh

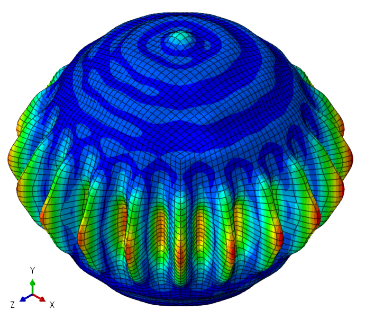

(d) Mode 7 with regular mesh

Figure 4: LBA - Complete sphere under external pressure: Different buckling modes obtained with the two types of mesh ure 2(b) . Indeed, even if the available experimental results of complete spheres subjected to external pressure show axisymmetric post-buckled patterns, at least on the onset of buckling [11], one knows from Koiter [7] and Hutchinson [4] that the fundamental theoretical buckling pattern for the perfect sphere is non-axisymmetric. Moreover, the theoretical chess-board mode can be obtained experimentally by limiting the development of buckling waves, using an inside mandrel (see Figure 5).

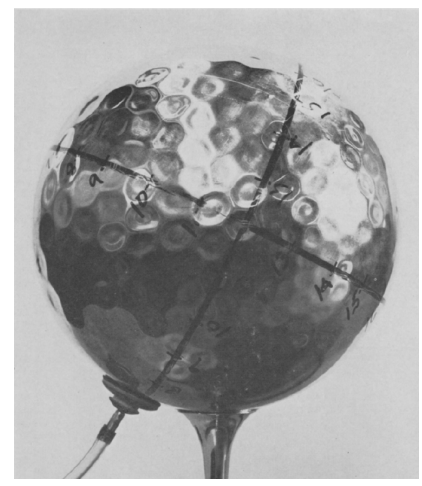

Figure 5: Nearly perfect electroformed spherical shell under external pressure [41] - in this picture, the post-buckling pattern is "artificially" stabilized because there is a solid mandrel inside the shell (adapted from [11])

\subsubsection{Materially Non-linear Analysis (MNA)}

Secondly, incremental calculations are performed (in small strains), considering here the material non-linearities. The constitutive law is assumed to be elastic/perfectly plastic, obeying the von Mises criterion in the context of the associated flow theory of plasticity. Due to perfect plasticity (namely, in the absence of strain hardening), a limit load is supposed to be asymptotically reached which corresponds to the reference MNA plastic collapse load $\left(P_{M N A}\right)$. The pressure-displacement curves in Figure 6(a) confirm this, as one observes invariably a final plateau at the collapse load for any geometric ratio $R / t$. Besides, Figure $6(\mathrm{~b})$ shows that the numerical collapse loads $P_{M N A}$ obtained here are 
consistent with the values given by the expression implemented in the ECCS text for the complete spherical shell under external pressure:

$$
P_{M N A}^{E C C S}=\sigma_{y}\left(\frac{2 t}{R}\right)
$$

This expression will thus be used in the sequel for the estimation of the plastic collapse load of a spherical shell under external pressure.

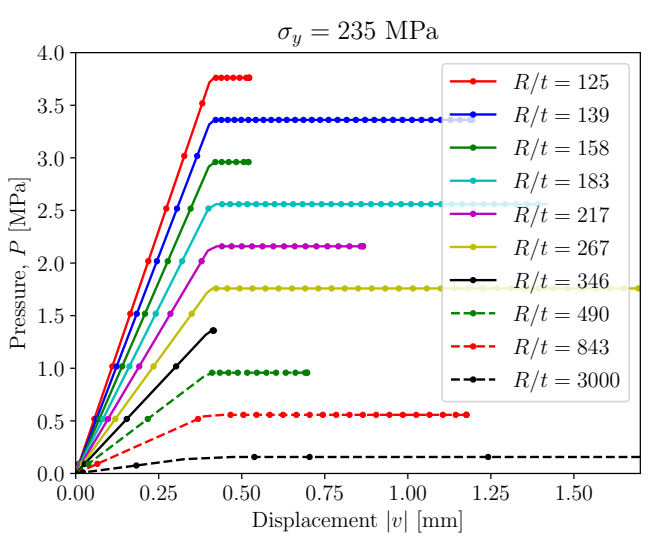

(a)

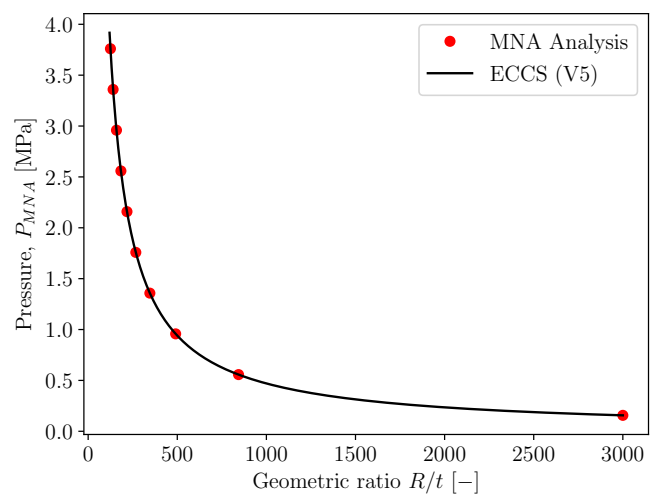

(b)

Figure 6: MNA - Complete spherical shell under external pressure: (a) Pressure vs. a characteristic displacement $v$ for different geometric ratios; (b) Comparison of the present numerical values with Equation (7)

\subsubsection{Geometrically Non-linear Analysis (GNA)}

As mentioned in Section 4, a bespoke FE program [39] is then used to study the non-linear buckling and postbuckling behavior of the perfect spherical shell under external pressure. In this context, for efficiency purposes, only one eighth of the spherical shell is modeled and the computations are carried out by taking into account all the relevant symmetry conditions (later, the imperfections used will be in the form of a symmetric mode so that this GNA on an eighth of the spherical shell is consistent with the subsequent analyses). The geometric non-linearities (in presence of large deflections) are overcome by means of a generalized arc-length procedure [38] and special "branch-switching" techniques. In elasticity, the critical pressures obtained for both cases of a follower and dead load are very close to $P_{c r_{(\text {Zoelly })}}=16.26 \mathrm{MPa}$ (with a maximum error of $1 \%$ ). Figure 7 represents the normalized pressure $P / P_{c r_{(Z o e l l y)}}$ versus the relative volume variation $\Delta V / V$ in both loading cases. One can observe that, just after the bifurcation point, the carrying capacity of the shell drops very sharply (in the form of a very sharp snap-back), which is characteristic of very imperfection-sensitive structures, as emphasized before. In Figure 8, successive post-critical deformed shapes corresponding to the follower pressure case are depicted. Just after the bifurcation point (Figure 8(a) $)$, the deformation pattern is reticulated, similarly to the linearized buckling mode previously obtained with ABAQUS. As one moves forward to the advanced post-buckling range (Figures $8(\mathrm{~b}), 8(\mathrm{c})$ and $8(\mathrm{~d})$, the deformation localizes in a single dimple, 
as already outlined by Hutchinson [6].

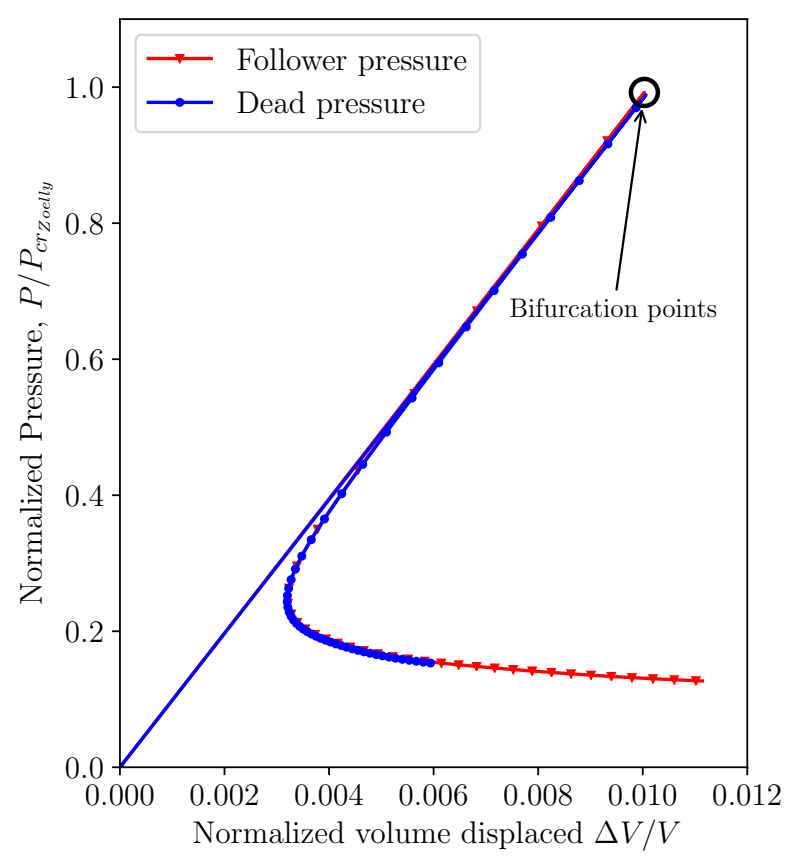

Figure 7: GNA - Complete sphere under external pressure: Normalized pressure $P / P_{c r_{\text {Zoelly }}}$ vs. normalized displaced volume $\Delta V / V$

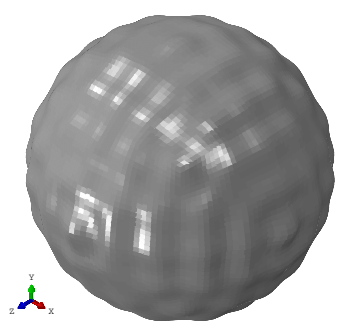

(a) Inc. BIF, $\times 1000$

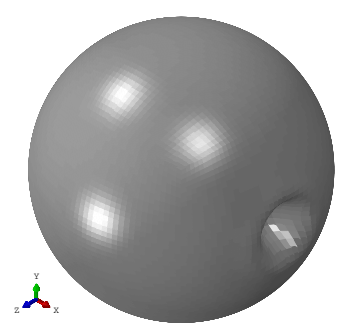

(b) Inc. $2624, \times 5$

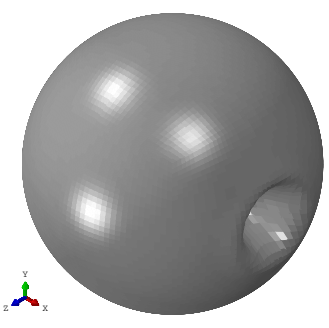

(c) Inc. $4270, \times 5$

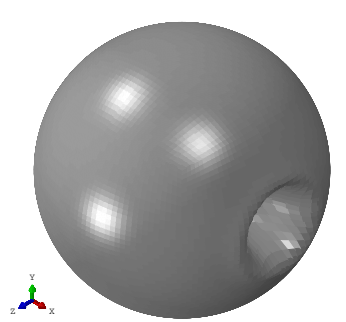

(d) Inc. $5577, \times 5$

Figure 8: GNA - Complete sphere under external pressure: Successive post-buckled deformation shapes in the more realistic follower pressure case

\subsubsection{Geometrically (and Materially) Non-linear Imperfect Analyses (GNIA and GMNIA)}

Further computations are then performed, considering initial geometric imperfections, so as to estimate the imperfection sensitivity of such a problem. The first linearized buckling mode previously obtained serves as imperfection pattern. The incremental calculations are completed using ABAQUS again. In the first instance, only geometric nonlinearities are taken into account (GNIA). Figure 9 represents the normalized pressure $P / P_{L B A}$ versus a characteristic displacement $v$ for several imperfection amplitudes $\delta_{0}$. One can observe that even a small imperfection amplitude can reduce significantly the ultimate elastic strength of the structure. Then, as the imperfection amplitude increases, the 
limit load continues to decline until stabilization is reached for very large defects. In these extreme cases, there is no longer truly a limit load. One can observe also that for $\delta_{0} / t>1.1$, the limit loads obtained are practically identical. These observations are consistent with the results of Lee et al. [18] who, by plotting $P_{G N I A} / P_{c r_{(Z o e l l y)}}$ against $\delta_{0} / t$, suggest the existence of a plateau after a certain value of imperfection amplitude (namely, for $\delta_{0} / t>1$ ).

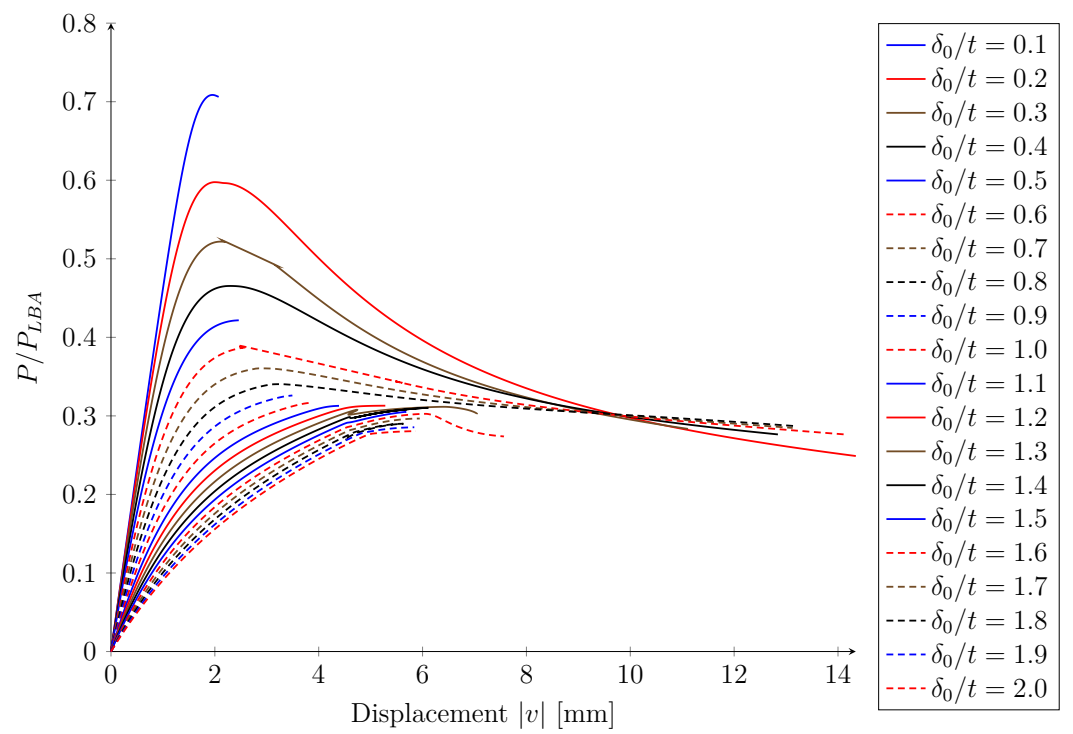

Figure 9: GNIA - Complete spherical shell under external pressure: Normalized pressure $P / P_{L B A}$ vs. a characteristic displacement $v$ for different imperfection amplitudes $\delta_{0}$

Figure 10] shows the post-critical deformation shapes obtained for different imperfection amplitudes in the advanced post-buckling range. One can remark that several dimples are randomly generated on the spherical shell, stemming from the localization of the diamond pattern observed both in the first linearized buckling mode acting as the imperfection shape $($ Figure $4(\mathrm{c})]$ and in the immediate post-critical deformation shape $($ Figure $8(\mathrm{a})]$. This behavior has been consistently observed for different geometric parameters $R / t$, with various numbers and sizes of dimples from case to case.

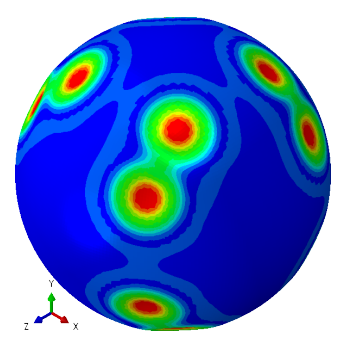

(a) $\delta_{0} / t=0.025$

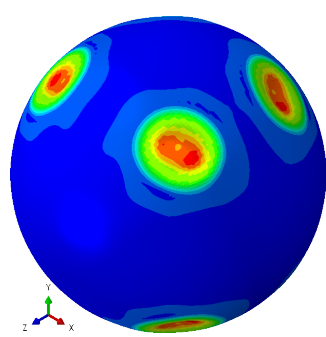

(b) $\delta_{0} / t=0.275$

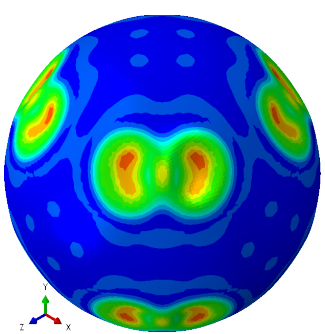

(c) $\delta_{0} / t=0.5$

Figure 10: GNIA - Complete spherical shell under external pressure: Post-critical deformation shapes for selected imperfection amplitudes in the advanced post-buckling range

According to the ECCS framework [30] and the European standard EN 1993-1-6 [31], the most comprehensive 
analysis which can be undertaken for the design of shell structures is a Geometrically and Materially Non-linear Analysis with explicit geometric Imperfections (GMNIA). Such an analysis takes into account both geometric and material non-linearities. The material used here is assumed again to follow an ideal elastic-plastic stress-strain law. Very recently, Zhang et al. [14] have shown that such an elastic-perfectly plastic material model led, for the spherical shell under external pressure, to an accurate match between numerical and experimental results. As previously, the buckling mode associated with the lowest critical pressure $P_{L B A}$ is set to be the initial geometric imperfection pattern. Figure 11 displays again the normalized pressure $P / P_{L B A}$ versus a characteristic displacement $v$ for different imperfection amplitudes $\delta_{0}$. One can observe that the reduction of the bearing capacity induced by very small imperfection amplitudes is still far more severe. However, as observed in the GNIA results, as soon as $\delta_{0}$ attains a particular value (say $\delta_{0} / t \geq 1.3$ ), the limit load no longer varies. Overall, the pressure levels reached in presence of plasticity are very small compared to the ones previously retrieved in the purely elastic GNIA parametric studies. Actually, for the geometric ratio considered $(R / t=125)$, plastic yielding occurs before buckling, hence the loss of stiffness which is highly responsible for this reduction of the bearing capacity of the shell.

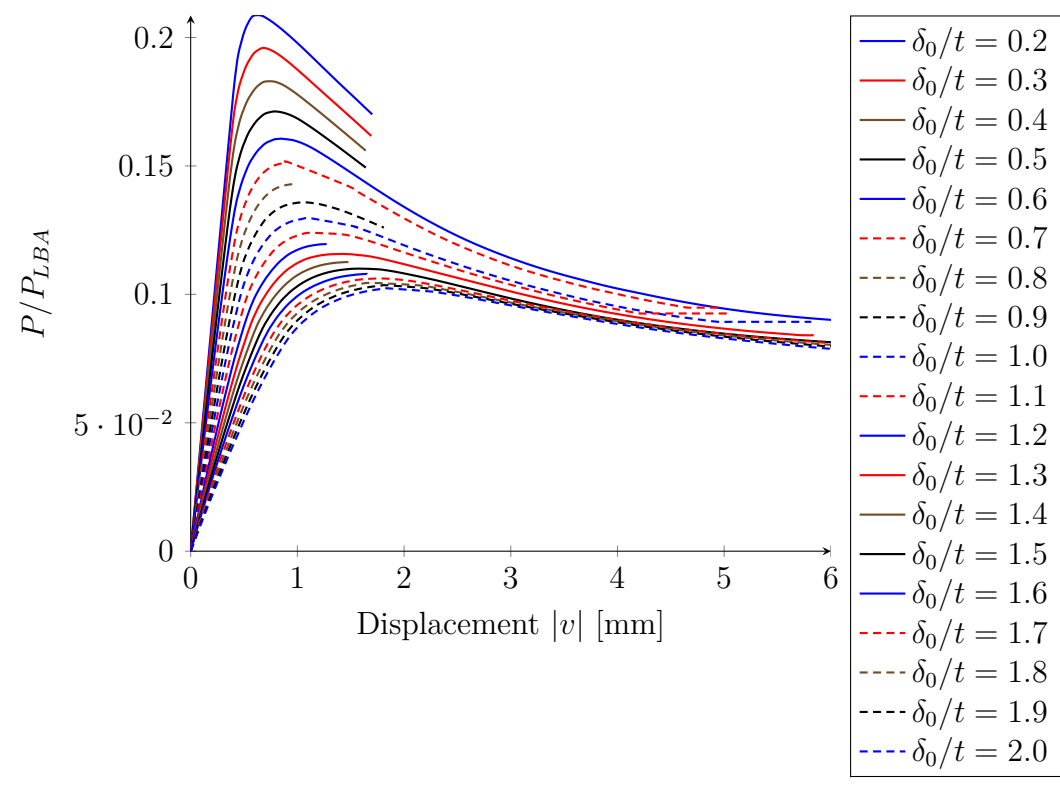

Figure 11: GMNIA - Complete spherical shell under external pressure: Normalized pressure $P / P_{L B A}$ vs. a characteristic displacement $v$ for different imperfection amplitudes $\delta_{0}$

\subsection{Buckling capacity curves}

As outlined in Section 2 , the Rotter's modified curves have been used in this study for extracting the key parameters of the buckling capacity curves. In the ECCS framework, the previous computations performed for several geometric ratios $R / t$ and imperfection amplitudes $\delta_{0}$ lead up to the definition of so many capacity curves. As an illustration, Figures 12(a) and 12(b) describe respectively the traditional and modified capacity curves obtained for $R / t=125$. 


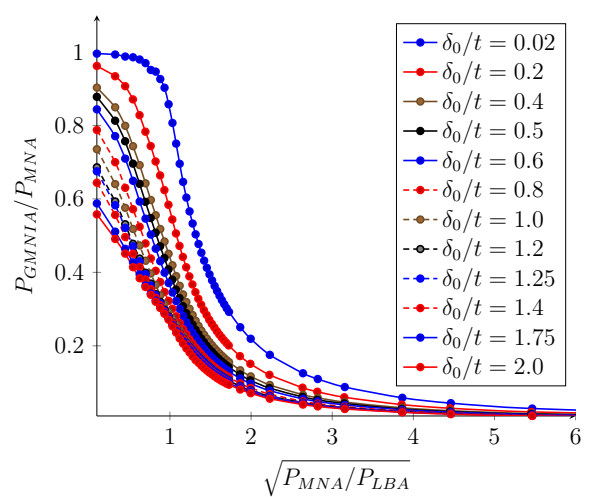

(a) Traditional capacity curve

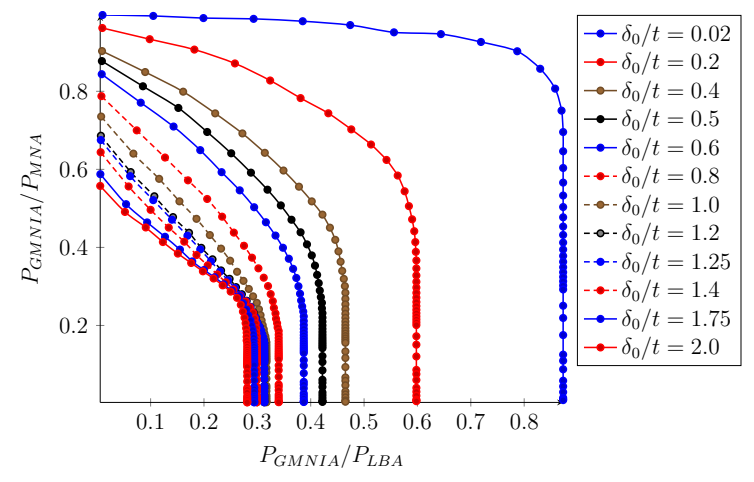

(b) Modified capacity curve

Figure 12: Complete spherical shell under external pressure: Buckling capacity curves for $R / t=125$

First of all, it should be pointed out that, since no strain hardening is intended (due to the perfectly plastic model), the squash limit is supposed all along to be $\lambda_{0}=0$ (it means that the extraction of the key parameters is performed under the assumption that all the resistances $R_{i}$ are related to elastic or elastoplastic buckling, and not to plastic breakdown). Besides, parameters $\alpha, \beta$ and $\eta$ are retrieved, thereby fitting the numerical curves by means of a non-linear least square scheme, and closed-form empirical expressions are obtained which actually depend only on $\delta_{0} / t$, after having retained the more conservative (lower bound) values with respect to the geometric ratio $R / t$.

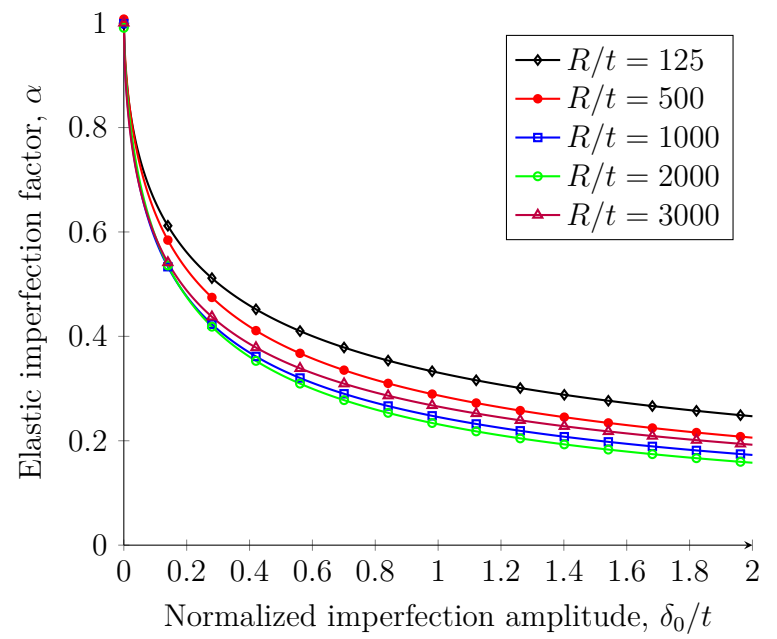

(a)

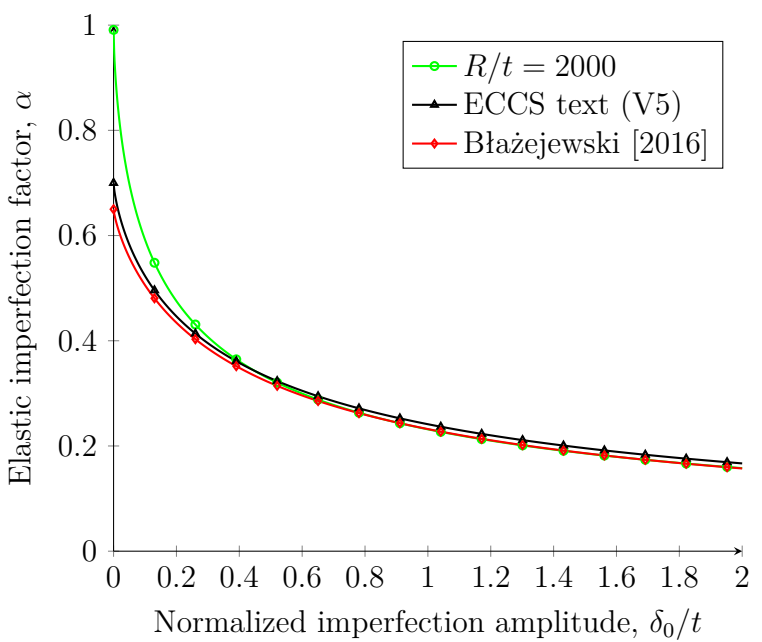

(b)

Figure 13: Complete spherical shell under external pressure: (a) Elastic imperfection factor $\alpha$ for $R / t=125,500,1000,2000,3000$; (b) Comparison of the present values of $\alpha$ with the values currently implemented in the ECCS text and the factors given by Błażejewski and Marcinowski [16

Figure 13 displays the elastic imperfection factor $\alpha$ versus the normalized imperfection amplitude $\delta_{0} / t$ for geometric ratios $R / t$ ranged between 125 and 3000. The evolution of $\alpha$ with respect to the ratio $R / t$ [Figure 13(a)] is probably due to the effect of the pre-buckling deformations which may have a strong influence on the elastic imperfection factor. 
For this reason, the designation "elastic imperfection factor" can be misleading, and it will be changed in the future provisions of the EN 1993-1-6 [31] by explicitly splitting the current factor into two factors which respectively take into account the influence of geometric non-linearities and geometric imperfections [42]. Here, the lowest factor $\alpha$ obtained from the parametric studies will be retained as a lower bound for the definition of the elastic imperfection factor. The comparison of the present factor $\alpha$ with other ones found in the literature is very satisfactory (see Figure 13(b)]. It appears that, for small imperfection amplitudes $\left(\delta_{0} / t<0.4\right)$, the factor $\alpha$ currently implemented in the ECCS text [30], the one recently given by Błażejewski and Marcinowski [16] and the present result deviate from each other. However, for $\delta_{0} / t \geq 0.4$, the lower bound curve defined here (corresponding to $R / t=2000$ ) fits perfectly the curve from Błażejewski and Marcinowski. A careful look at the paper from these authors shows that their expression given for $\alpha$ has only been defined from numerical points with $\delta_{0} / t>0.4$, hence the deviation observed for small imperfection amplitudes.

Furthermore, it must be highlighted that conservative constant values have been retained for parameters $\beta$ and $\eta$ (namely, $\beta=0.70$ and $\eta=1.0$ ) to be implemented in the ECCS text. Drawing a parallel with the case of a cylindrical shell under axial compression [43], one can expect to find more precise results for both parameters, considering that enough results are available. Recently, Błażejewski and Marcinowksi [16] have derived an explicit expression of parameter $\beta$ (for a clamped spherical cap) which depends on the normalized imperfection amplitude $\delta_{0} / t$ :

$$
\beta=0.87 \cdot\left(\frac{\delta_{0}}{t}\right)^{0.026}
$$

Equation (8) shows that the constant value of $\beta$ currently adopted in the ECCS text is only conservative for certain values of the imperfection amplitude. Here, the previous parametric studies allow us to extract and define the parameters $\beta$ and $\eta$ as closed-form expressions which also depend on the normalized imperfection amplitude $\delta_{0} / t$. These expressions write as follows:

$$
\beta=1-\frac{a_{1}}{1+a_{2} \cdot\left(\frac{\delta_{0}}{t}\right)^{a_{3}}} \quad \eta=a_{4}+\frac{a_{5}}{a_{6}+\left(\frac{\delta_{0}}{t}\right)}
$$

with coefficients $a_{i}$ listed in Table 3 A lower bound expression of $\beta$ can also be derived by taking the maximum value obtained for $a_{2}$.

\begin{tabular}{rcccccc}
\hline$R / t$ & $a_{1}$ & $a_{2}$ & $a_{3}$ & $a_{4}$ & $a_{5}$ & $a_{6}$ \\
\hline 125 & 1 & 4.58 & 0.59 & 0.016 & 0.679 & 0.201 \\
500 & 1 & 6.54 & 0.58 & 0.278 & 0.150 & 0.018 \\
1000 & 1 & 8.14 & 0.57 & 0.177 & 0.189 & 0.037 \\
\hline
\end{tabular}

Table 3: Plastic range factor $\beta$ and interaction exponent $\eta$ : Parameters $a_{1}, a_{2}, a_{3}, a_{4}, a_{5}$ and $a_{6}$ obtained by interpolation for $R / t=125,500,1000$ 


\section{Spherical shell under circumferential shear} terms.

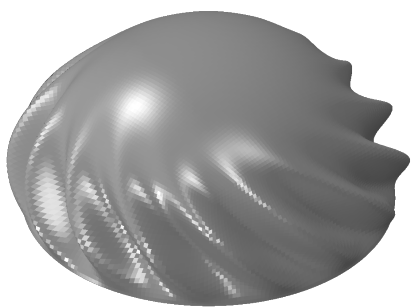

(a) $Q_{L B A}=20.097 \mathrm{MPa}$

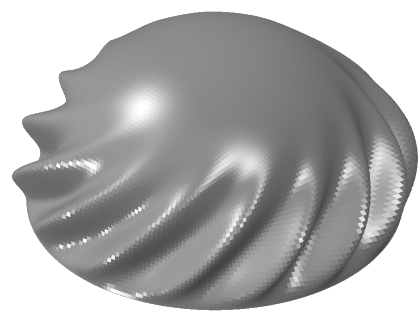

(b) $Q_{L B A}=-20.097 \mathrm{MPa}$

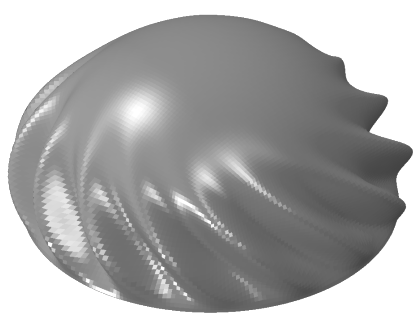

(c) $Q_{L B A}=20.097 \mathrm{MPa}$

Figure 14: LBA - Spherical shell under circumferential shear: First three linearized buckling modes (the number of critical buckling waves $n_{c r}$ is equal to 14 for these first three modes)

The buckling response of shells is frequently affected by the pre-buckling deformations, which may impinge on the buckling mode or the bearing capacity of the shells. In the case of a spherical shell under circumferential shear load, many convergence difficulties are systematically encountered when dealing with Geometrically Non-linear Analyses (using ABAQUS) despite the application of arc-length methods [38]. Two methods can be used to overcome those issues:

- One can "degenerate" the problem by introducing an imperfection scaled with a very small amplitude. The introduced imperfection distorts then the pre-buckling solution in such a way that the bifurcation point disappears and converts the buckling problem into a more regular problem only involving a possible limit point.

- One can use the so-called stabilization method, for unstable quasi-static problems, of ABAQUS/Standard [37]. It consists in the automatic addition of volume-proportional damping to the model [44, 45]. This latter method is used here so as to achieve convergence near the bifurcation point. For the sake of completeness, let us mention 
that the damping factor applied in our analysis is chosen to be equal to $4 \cdot 10^{-4}$ and that the accuracy tolerance used for the adaptive stabilization scheme is 0.05 (default value).

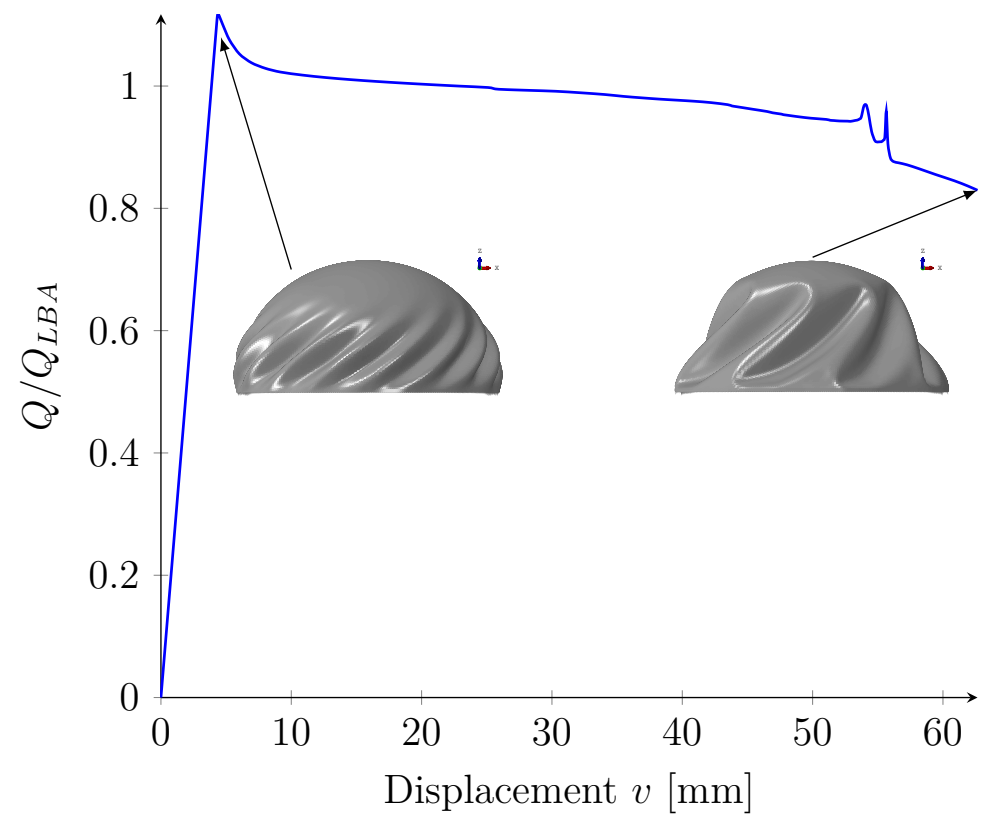

Figure 15: GNA - Spherical shell under circumferential shear: Normalized buckling load $Q / Q_{L B A}$ vs. a characteristic displacement $v$ (the Artificial Damping Method is used with a damping factor of 1/2500)

The post-buckling path of the spherical shell under circumferential shear (reference model) is fully described in Figure 15. After the limit point (which almost coincides with the bifurcation point), the post-critical branch reaches something like a plateau (with a very soft negative slope). Therefore, the problem of a spherical shell under circumferential shear is probably less imperfection-sensitive than the one of a spherical shell under external pressure. Just after the bifurcation point, the deformation shape is quite similar to the one obtained with LBA (Figure 14) with a slightly greater wave number $\left(n_{c r}=16\right)$. In the advanced post-buckling range, the size of the buckling waves increases and, paradoxically, their numbers decrease. It is likely that some of the buckling waves which are generated at the bifurcation point progressively merge with each other.

\subsubsection{GNIA and GMNIA}

The analysis of the imperfect structure is carried out by setting the eigenmode associated with the lowest critical buckling load $Q_{L B A}$ as imperfection pattern. Parametric studies are performed by varying the imperfection amplitude $\delta_{0}$, what leads up to the different equilibrium paths displayed in Figure 16.

As seen in Figure 16(a), the reduction of the limit load with the imperfection amplitude is slightly less pronounced here than in the previous loading case of external pressure. Moreover, in most cases, the limit load totally vanishes and the incremental curve appears to be regular (monotonous), highlighting thus a certain reserve in the post-buckling range. As far as plasticity is concerned, the previous plateau and relative stability of the post-critical behavior gradually 


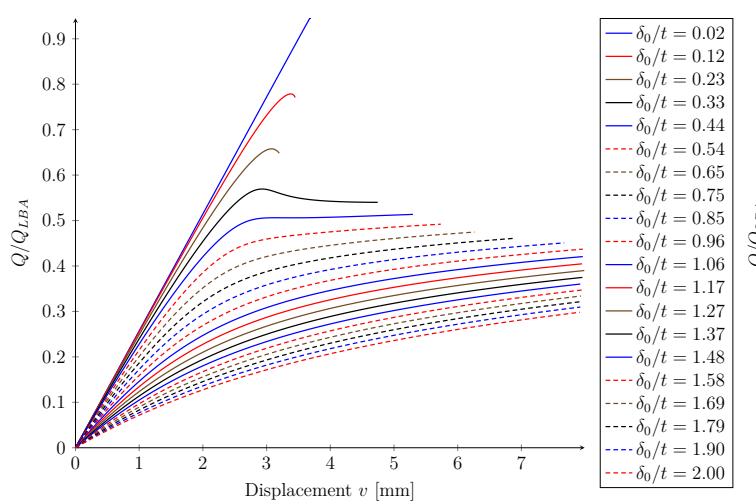

(a) $\operatorname{GNIA}(R / t=125)$

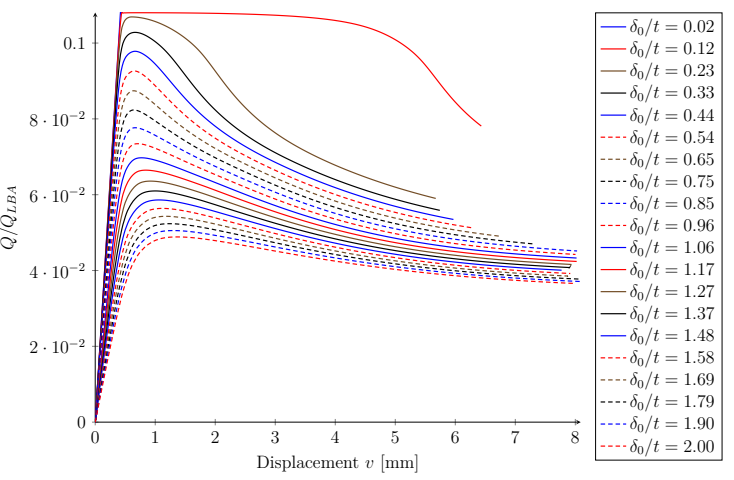

(b) GMNIA $(R / t=125)$

Figure 16: GNIA/GMNIA - Spherical shell under circumferential shear: Normalized load $Q / Q_{L B A}$ vs. a characteristic displacement $v$

disappears when increasing the imperfection amplitude (see Figure 16(b)]. The critical loads are extremely low compared to the ones obtained in elasticity (GNIA) and the bifurcation/limit points are followed by a sharp decline. For the particular geometric ratio of the reference model $(R / t=125)$, as in the case of external pressure, yielding precedes buckling. As a consequence, the loss of stiffness combined to the effect of geometric imperfections reduces considerably the bearing capacity of the shell.

\subsection{Buckling capacity curves}

As done in Section 5.2 the previous parametric studies at the different "computation levels" of the ECCS text allow us to build the so-called buckling capacity curves for a spherical shell under circumferential shear. From these capacity curves, the key parameters $\alpha, \beta$ and $\eta$ can be retrieved, something that was unprecedented, to the best knowledge of the authors. For illustrative purposes, Figure 17 shows the elastic imperfection factor $\alpha$ for different geometric ratios and the corresponding lower bound (as a function representing the best fit of the numerical data) versus the normalized imperfection amplitude $\delta_{0} / t$.

Again, the different values of $\alpha$ are almost identical whatever the geometric ratio $R / t$. Since $\alpha$ reflects the influence of geometric non-linearities and geometric imperfections, the pre-buckling deformations may have here a little influence on the global response in this configuration. For monitoring purposes, the circumferential displacement (Figure 18(a) and the shear stress (Figure 18(b) along a meridian of the spherical shell are plotted in both cases of a Linear Analysis (LA) and a Geometrically Non-linear Analysis (GNA) (just before the bifurcation point). One can easily check that there are no significant differences between the values obtained with the two different analyses, evidence that the geometric non-linearities do not play any role during the pre-buckling stage.

\section{Spherical shell under combined loading}

The final scope of this paper is to deal with the buckling behavior of spherical shells under combined loadings, namely external pressure and circumferential shear loads. In the previous sections, the buckling behavior of a spherical 


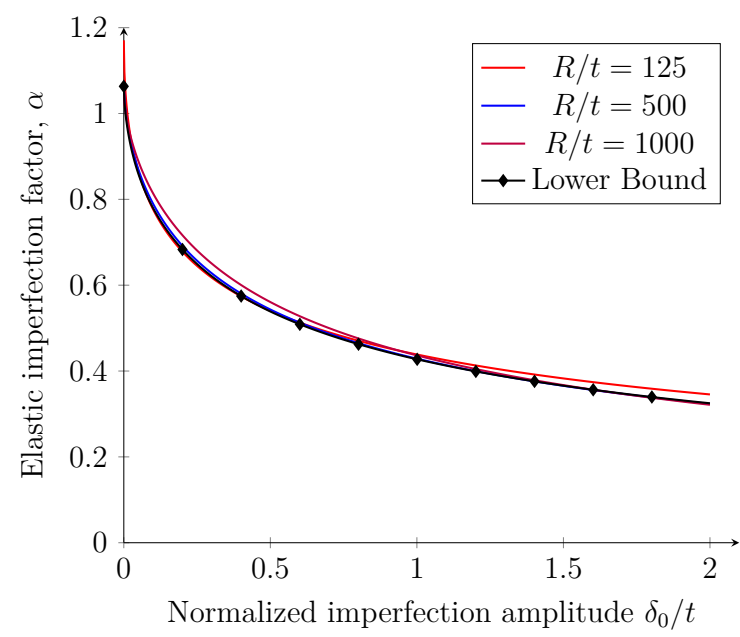

Figure 17: Spherical shell under circumferential shear: Elastic imperfection factor $\alpha$ for different geometric ratios $R / t$

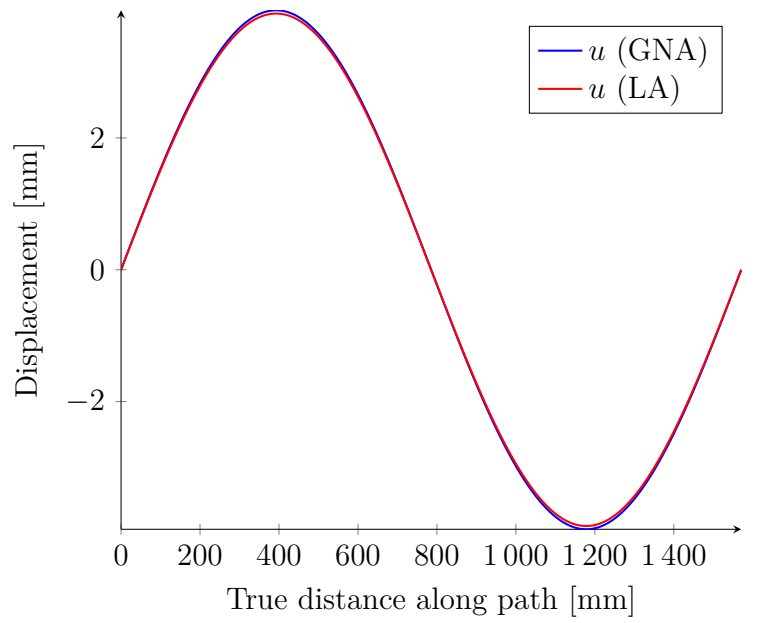

(a) Displacement $u$

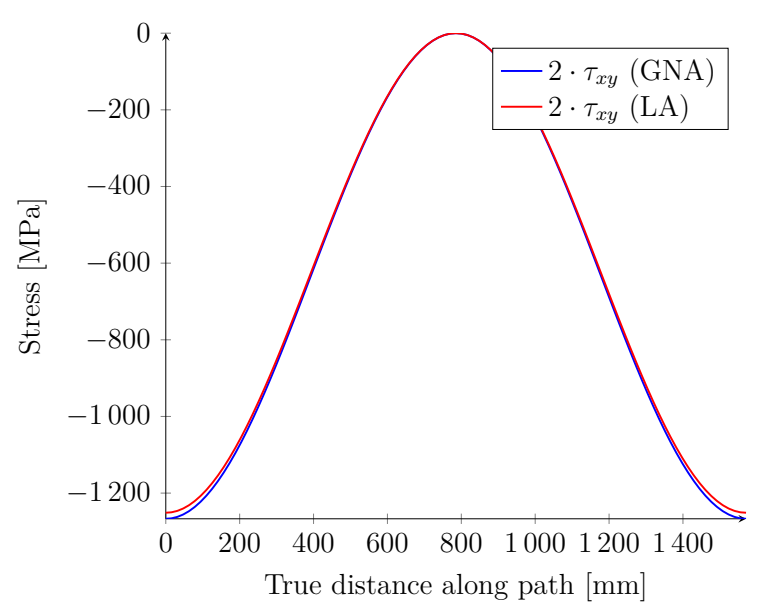

(b) Stress $\tau_{x y}$

Figure 18: Spherical shell under circumferential shear: Displacement $u$ and stress $\tau_{x y}$ along a meridian for a Linear Analysis (LA) and just before the bifurcation point in a Geometrically Non-linear Analysis (GNA)

shell under the two fundamental load cases has been highlighted. Henceforth, in the case of a combination of loads, the stress distribution in the pre-buckling state may also be a combination of the simple ones associated to the respective fundamental loads. Thus, it is natural to put underway reliable methods in order to verify, for shell structures subjected to complex load combinations, the safety against buckling. As mentioned in Section 1. Schmidt and Winterstetter [21] have already derived from numerous parametric studies an interaction rule for cylindrical shells under combined loadings which is currently implemented in the ECCS text [30] and in the EN 1993-1-6 [31]. This interaction rule has the particular feature to be represented by a straight line when buckling is purely elastic, and to follow asymptotically the von Mises yield surface (which may be obtained through a Materially Non-linear Analysis) when plastic breakdown occurs, with a transitional part where plasticity interacts with stability, giving rise to elastoplastic buckling. 
Analogously to Schmidt and Winsterstetter [21], parametric studies have been undertaken here for the spherical shell under combined external pressure and circumferential shear, with several geometric ratios $R / t$ for both perfect and imperfect geometries.

\subsection{Perfect shell}

First of all, elastic Linear Bifurcation Analyses are performed for several loading combinations varying from a pure external pressure to a pure circumferential shear. In each case, the bifurcation point (critical pressure vs. critical shear load) is plotted so as to draw the so-called interaction curves in Figure 19(a) The shapes of the interaction curves observed here are very consistent with the pioneering work of Mow and Sadowski [20] on the buckling of spherical shells subjected to a moment torque and external (or internal) pressure. Generally speaking, the interactive buckling curves are only slightly dependent on slenderness, in a large range of geometric ratios $R / t$. For thick shells (namely, low values of $R / t$ ), the interactive curves display a parabolic part in the range of low shear-to-pressure ratios. As one increases the shear ratio $Q / Q_{L B A}$, the interaction curves become almost straight. In contrast, for thinner shells (namely, high values of $R / t$ ), the interaction curves are perfectly straight all along. Proof of this are the exponents $\gamma_{1}$ and $\gamma_{2}$ retrieved from the best fit of the interaction curves (see the reference equation in Figure 19(a)), which are represented in Figure 19(b) and take almost invariably a value close to unity.

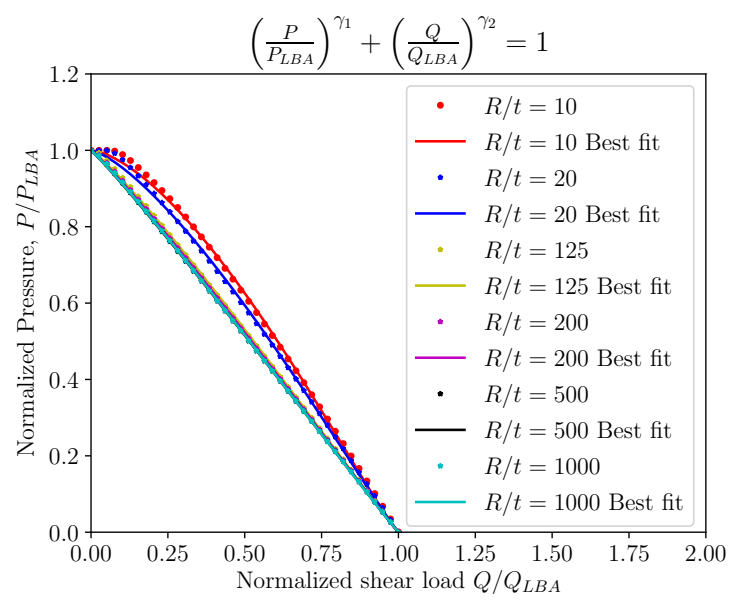

(a)

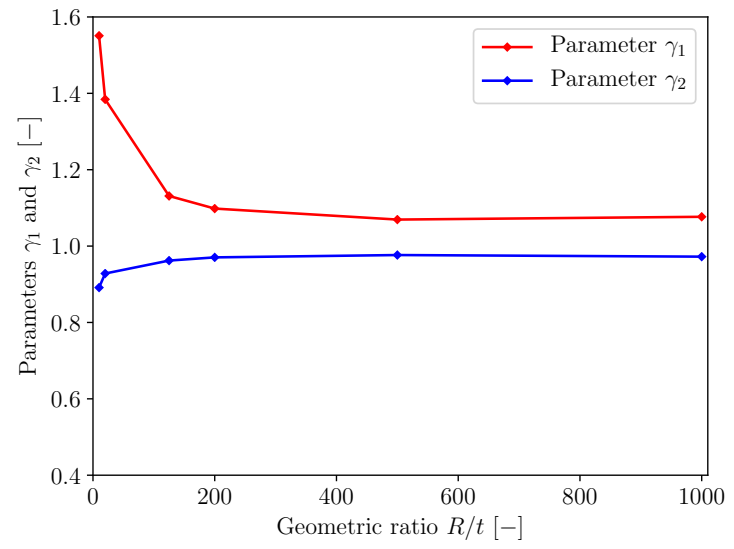

(b)

Figure 19: Spherical shell under combined loading: (a) Interactive buckling curves using LBA for several geometric ratios; (b) Parameters $\gamma_{1}$ and $\gamma_{2}$ retrieved by fitting the numerical curves using the equation $\left(P / P_{L B A}\right)^{\gamma_{1}}+\left(Q / Q_{L B A}\right)^{\gamma_{2}}=1$

Geometrically (and Materially) Non-linear Analyses have also been carried out in order to highlight the possible influence of pre-buckling deformations and material non-linearities. Figure 20 represents the interactive buckling curves arising from different analysis levels (LBA, GNA, GMNA) for $R / t=200$ and $R / t=500$. The interactive curves obtained in the GNA case fit perfectly the ones derived in the LBA case (since the bifurcation and limit loads are almost coincident). Only minor deviations have been noticed for a large range of geometric ratios. Therefore, it seems that the pre-buckling deformations have no particular effect on the buckling behavior under combined loading. 
In the GMNA case, the interactive buckling curves appear to be more slenderness-dependent than before. Indeed, with $R / t=200$, when the limit load is governed by plastic breakdown, the GMNA interactive curve perfectly coincides with the so-called von Mises yield surface, which is defined by the couples of loadings at which plastic collapse occurs. Conversely, with $R / t=500$, three different behaviors may happen (see Figure 20(b) : (i) elastic buckling (the interactive curve fits perfectly the ones retrieved from the LBA or GNA cases), (ii) plastic breakdown (the curve fits the von Mises yield surface) and (iii) elastoplastic buckling (corresponding to the transitional part of the curve between the two previous limit cases).

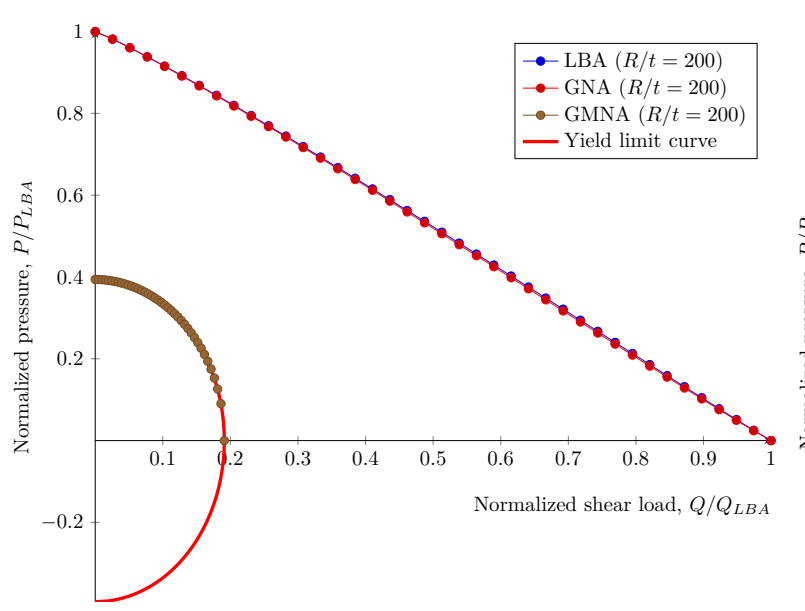

(a) $R / t=200$

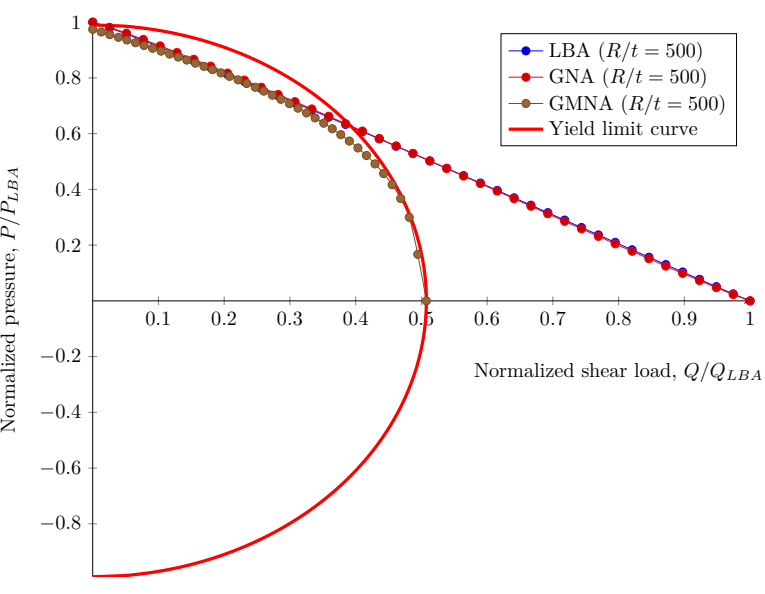

(b) $R / t=500$

Figure 20: Interactive buckling curves of a perfect spherical shell under combined loading $\left(E=210 \mathrm{GPa}, v=0.3, \sigma_{y}=250 \mathrm{MPa}\right)$

\subsection{Imperfect shell}

In order to study the general behavior of an imperfect spherical shell under combined loading, use is made of an imperfection pattern in the form of the eigenmode associated to the lowest buckling pressure $P_{L B A}$ of the spherical shell under external pressure only. For the sake of generality, several imperfection amplitudes $\delta_{0}$ have been considered.

The numerical results show that the corresponding interactive curves highly depend on the imperfection amplitude $\delta_{0}$ and are still slenderness-dependent. The following observations can be made:

- When buckling occurs in the elastic range, the GMNIA curves follow the ones obtained in the LBA/GNA cases. Conversely, when plastic breakdown precedes buckling, the GMNIA curves follow asymptotically the von Mises yield surface.

- The imperfection amplitude mainly contributes to reduce the safety domain of the interactive curves.

- It is noteworthy that for the imperfect spherical shell subjected to external pressure and circumferential shear, there exists a shear threshold below which the shear load has no effect on the combined load as seen in Figure 21 However, when this threshold is exceeded, the interaction becomes slenderness-dependent again. 


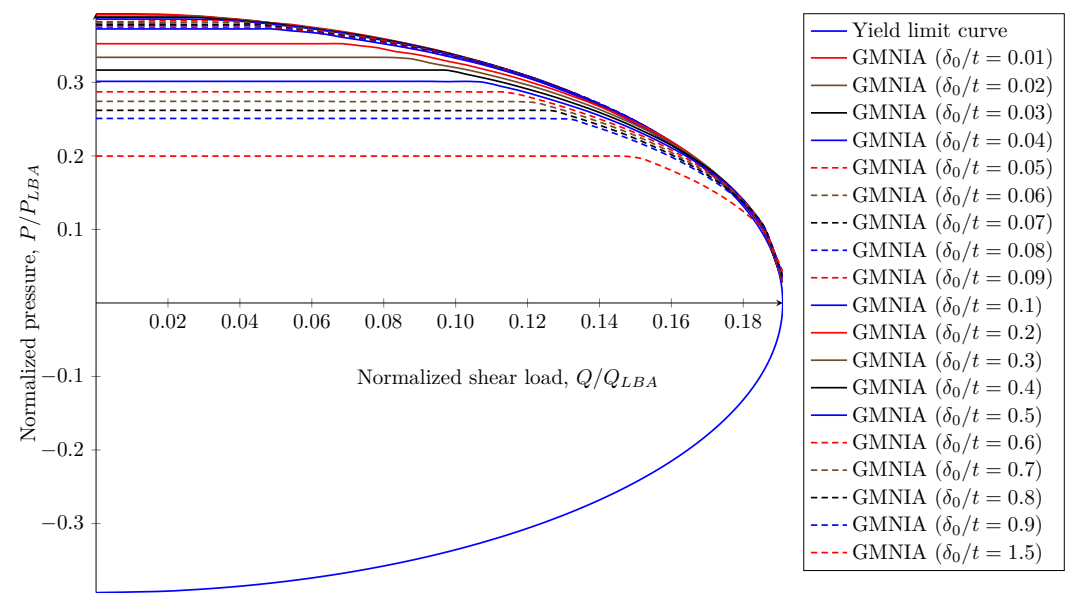

Figure 21: Interactive buckling curves of an imperfect spherical shell under combined loading $\left(R / t=200, E=210 \mathrm{GPa}, v=0.3, \sigma_{y}=250 \mathrm{MPa}\right)$

A perspective of this work, which is beyond the scope of this paper, would be to build an interaction rule based on the different interaction curves above. As emphasized before, the interaction curves generally depend on the actual imperfection amplitude and are slenderness-dependent. Thus, the interaction rule will be of the same kind than the one implemented in the ECCS text for the cylindrical shells and it will naturally depend on the different key parameters $(\alpha$, $\beta, \eta$ and $\lambda_{0}$ ) of the buckling capacity curves.

\section{Conclusion}

This paper is devoted to the analysis of the buckling behavior of spherical shell structures under external pressure and circumferential shear. Using the different "computations levels" of the ECCS text [30] and the EN 1993-1-6 [31], the buckling capacity curves and the related key parameters $\alpha, \beta$ and $\eta$ have been fully defined for both individual loadings. While more precise definitions of parameters $\beta$ and $\eta$ have been originally proposed for the external pressure case, the whole procedure applied to the circumferential shear case and especially the resultant buckling capacity curves are clearly innovative, to the best knowledge of the authors. Furthermore, the interactive buckling curves retrieved from the numerous parametric computations performed emphasize the slenderness dependency of the buckling behavior of spherical shell structures under combined pressure and circumferential shear. In a qualitative manner, at least for the perfect shell and in the linear case, the present interaction curves look like the ones given by Mow and Sadowski [20]. For the imperfect shell, the global behavior and the collapse load are also shown to be highly dependent on the normalized imperfection amplitude $\delta_{0} / t$, which mainly contributes to reduce the safety domain of the interactive buckling curves. On the basis of the promising findings presented in this paper, the more general and practical case of spherical caps will be investigated in our future works. 


\section{Acknowledgments}

The first author was funded by a CIFRE grant from the French technical center CETIM subsidized by the ANRT (convention number 2014/1177). The support of Mines Douai Engineering School is also gratefully acknowledged.

[1] W. T. Koiter, Over de Stabiliteit van het elastisch Evenwicht (On the stability of elastic equilibrium), Ph.D. thesis, Delft University of Technology, [English translations: NASA TT-F10 (1967) and AFFDL TR-7025 (1970)] (1945).

[2] R. Zoelly, Ueber ein Knickungsproblem an der Kugelschale, Ph.D. thesis, Eidgenössische Technische Hochschule Zürich (1915).

[3] A. Van der Neut, De elastische Stabiliteit van den dunwandigen Bol, Ph.D. thesis, Delft University of Technology (1932).

[4] J. W. Hutchinson, Imperfection sensitivity of externally pressurized spherical shells, Journal of Applied Mechanics 34 (1) (1967) 49-55.

[5] T. Koga, N. J. Hoff, The axisymmetric buckling of initially imperfect complete spherical shells, International Journal of Solids and Structures 5 (7) (1969) 679-697.

[6] J. W. Hutchinson, Buckling of spherical shells revisited, Proceedings of the Royal Society of London A: Mathematical, Physical and Engineering Sciences 472 (2195) (2016) 20160577.

[7] W. T. Koiter, The nonlinear buckling problem of a complete spherical shell under uniform external pressure, Proceedings of the Koninklijke Nederlandse Akademie van Wetenschappen, Series B-Physical Sciences 72 (1) (1969) 40-123.

[8] G. D. Galletly, J. Błachut, J. Kruzelecki, Plastic buckling of imperfect hemispherical shells subjected to external pressure, Proceedings of the Institution of Mechanical Engineers, Part C: Journal of Mechanical Engineering Science 201 (3) (1987) 153-170.

[9] G. D. Galletly, J. Błachut, Buckling design of imperfect welded hemispherical shells subjected to external pressure, Proceedings of the Institution of Mechanical Engineers, Part C: Journal of Mechanical Engineering Science 205 (3) (1991) 175-188.

[10] W. Wunderlich, U. Albertin, Buckling behaviour of imperfect spherical shells, International Journal of Non-Linear Mechanics 37 (4) (2002) 589-604.

[11] R. L. Carlson, R. L. Sendelbeck, N. J. Hoff, Experimental studies of the buckling of complete spherical shells, Experimental Mechanics 7 (7) (1967) 281-288.

[12] L. Berke, R. L. Carlson, Experimental studies of the postbuckling behavior of complete spherical shells, Experimental Mechanics 8 (12) (1968) 548-553

[13] P. R. Murray, Stability of spherical shells, Ph.D. thesis, University of Oxford (1978).

[14] Z. Jian, Z. Meng, T. Wenxian, W. Weibo, W. Minglu, Buckling of spherical shells subjected to external pressure: A comparison of experimental and theoretical data, Thin-Walled Structures 111 (2017) 58-64.

[15] M. Deml, W. Wunderlich, Direct evaluation of the "worst" imperfection shape in shell buckling, Computer Methods in Applied Mechanics and Engineering 149 (1) (1997) 201-222.

[16] P. Błażejewski, J. Marcinowski, Buckling capacity curves for pressurized spherical shells, in: Recent Progress in Steel and Composite Structures: Proceedings of the XIII International Conference on Metal Structures (ICMS2016, Zielona Góra, Poland, 15-17 June 2016), CRC Press, 2016, p. 401

[17] F. L. Jiménez, J. Marthelot, A. Lee, J. W. Hutchinson, P. M. Reis, Technical brief: Knockdown factor for the buckling of spherical shells containing large-amplitude geometric defects, Journal of Applied Mechanics 84 (3) (2017) 034501.

[18] A. Lee, F. L. Jiménez, J. Marthelot, J. W. Hutchinson, P. M. Reis, The geometric role of precisely engineered imperfections on the critical buckling load of spherical elastic shells, Journal of Applied Mechanics 83 (11) (2016) 111005.

[19] Z. Yinyi, Torsional buckling of spherical shells under circumferential shear loads, Applied Mathematics and Mechanics 20 (4) (1999) 426-432.

[20] C. C. Mow, M. A. Sadowski, Determination of the critical torque inducing buckling in a twisted spherical shell subject to internal or external pressure, in: 1962 Army Science Conference, United States Military Academy, West-Point, New York, 20-22 June 1962, Armed Services Technical Information Agency, Arlington Hall Station, Arlington 12, Virginia, 1962. 
[21] T. A. Winterstetter, H. Schmidt, Stability of circular cylindrical steel shells under combined loading, Thin-Walled Structures 40 (10) (2002) 893-910

[22] G. D. Galletly, K. Pemsing, Interactive buckling tests on cylindrical shells subjected to axial compression and external pressure - A comparison of experiment, theory and various codes, Proceedings of the Institution of Mechanical Engineers, Part C: Journal of Mechanical Engineering Science 199 (4) (1985) 259-280.

[23] J. Odland, Design codes for offshore structures, buckling of cylindrical shells, in: J. F. Jullien (Ed.), Buckling of Shell Structures, on Land, in the Sea and in the Air, Elsevier Applied Science, 1991, pp. 277-285.

[24] T. A. Winterstetter, H. Schmidt, Cylindrical shells under torsional and transverse shear, in: J. G. Teng, J. M. Rotter (Eds.), Buckling of Thin Metal Shells, 1st Edition, Taylor \& Francis, 2004, Ch. 8, pp. 207-229.

[25] A. Berkovits, J. Singer, T. Weller, Buckling of unstiffened conical shells under combined loading, SESA Spring Meeting (1967) $458-467$.

[26] O. F. Ifayefunmi, Combined stability of conical shells, Ph.D. thesis, University of Liverpool (2011).

[27] J. Błachut, D. Stanier, Elastic buckling of conical shells under combined loading of axial compression and external pressure, in: B. Topping (Ed.), Proceedings of the $11^{\text {th }}$ International Conference on Computational Structures Technology, Civil-Comp Press, Stirlingshire, UK, 2012, Paper 163.

[28] A. Evkin, M. Kolesnikov, D. A. Prikazchikov, Buckling of a spherical shell under external pressure and inward concentrated load: Asymptotic solution, Mathematics and Mechanics of Solids (2016) 1081286516635872.

[29] A. B. Sabir, Large deflection and buckling behaviour of a spherical shell with inward point load and uniform external pressure, Journal of Mechanical Engineering Science 6 (4) (1964) 394-404.

[30] J. M. Rotter, H. Schmidt, Stability of steel shells: European design recommendations (5 ${ }^{\text {th }}$ edition), Tech. rep., European Convention for Constructional Steel Works, Brussels, Belgium (2008).

[31] EN 1993-1-6, Eurocode 3: Design of steel structures, Part 1-6: Strength and Stability of Shell Structures, Tech. rep., Comité Européen de Normalisation, Brussels, Belgium (2007).

[32] J. M. Rotter, The philosophy of shell buckling design according to EN 1993-1-6, in: Proc. $5^{\text {th }}$ Int. Conf. on Computation of Shell and Spatial Structures, Salzburg, Austria, 2005.

[33] J. M. Rotter, The practical design of shell structures exploiting different methods of analysis, in: Shell Structures: Theory and Applications, W. Pietraszkiewicz \& C. Szymczak, Taylor \& Francis, London, 2005.

[34] J. M. Rotter, The elastic-plastic imperfection sensitivity of axially compressed cylinders with weld depressions, in: Proc. Eurosteel 2008 Conference, 2008, pp. 3-5.

[35] W. Wunderlich, U. Albertin, Analysis and load carrying behaviour of imperfection sensitive shells, International Journal for Numerical Methods in Engineering 47 (1-3) (2000) 255-273.

[36] C. Doerich, J. M. Rotter, Generalised capacity curves for stability and plasticity: Application and limitations, Thin-Walled Structures 49 (9) (2011) 1132-1140.

[37] Simulia, Abaqus/Standard 6.14 User's Manual, Dassault Systems, Providence, RI (2014).

[38] E. Riks, Some computational aspects of the stability analysis of nonlinear structures, Computer Methods in Applied Mechanics and Engineering 47 (3) (1984) 219-259.

[39] P. Le Grognec, A. Le van, Elastoplastic bifurcation and collapse of axially loaded cylindrical shells, International Journal of Solids and Structures 45 (1) (2008) 64-86.

[40] J. W. Hutchinson, Knockdown factors for buckling of cylindrical and spherical shells subject to reduced biaxial membrane stress, International Journal of Solids and Structures 47 (10) (2010) 1443-1448.

[41] J. M. T. Thompson, The elastic instability of spherical shells, Ph.D. thesis, Cambridge University (1961).

[42] J. M. Rotter, Shell buckling design and assessment and the LBA-MNA methodology, Stahlbau 80 (11) (2011) $791-803$.

[43] J. M. Rotter, The elastic-plastic imperfection sensitivity of axially compressed cylinders with weld depressions, in: Proc. Eurosteel 2008 
[44] T. Kobayashi, Y. Mihara, F. Fujii, Path-tracing analysis for post-buckling process of elastic cylindrical shells under axial compression, Thin-Walled Structures 61 (2012) 180-187.

[45] A. J. Sadowski, J. M. Rotter, Modelling and behaviour of cylindrical shell structures with helical features, Computers and Structures 133 (2014) 90-102. 